\title{
Minimum Cost Consensus Models based on Random Opinions
}

\author{
Ning Zhang ${ }^{\mathrm{a}}$, Zaiwu Gonga,*, Francisco Chiclana ${ }^{\mathrm{b}}$ \\ ${ }^{a}$ School of Economics and Management, Nanjing University of Information Science and Technology, Nanjing 210044, \\ China \\ ${ }^{b}$ Centre for Computational Intelligence, Faculty of Technology, De Montfort University, Leicester, UK
}

\begin{abstract}
In some complex group decision making cases, the opinions of decision makers (DMs) present random characteristic. However, it is difficult to determine the range of opinions by knowing only their probability distributions. In this paper, we construct cost consensus models with random opinions. The objective function is obtaining the minimum consensus budget under a certain confidence level. Nonetheless, the constraints restrict the upper limit of the consensus cost, the lower limit of DMs' compensations, and the opinions deviation between DMs and the moderator. As such, probabilistic planning based on a genetic algorithm is designed to resolve the minimum cost consensus models based on China's urban demolition negotiation, which can better simulate the consensus decisionmaking process and obtain a satisfactory solution for the random optimization consensus models. The proposed models generalize both Ben-Arieh's minimum cost consensus model and Gong's consensus model with uncertain opinions. Considering that the opinions of DMs and the moderator obey various distributions, the models simulate the opinion characteristics more effectively. In the case analysis, a sensitivity analysis method is adopted to obtain the minimum budget, and probabilistic planning based on genetic algorithm to obtain a satisfactory solution that is closer to reality.
\end{abstract}

Keywords: Group decision making, Consensus, Probability distribution, Probabilistic planning, Genetic algorithm.

\section{Introduction}

Group consensus is mainly used to analyze the consistency of group opinions. Four aspects of consensus research can be summarized as follows: the degree of consensus is related to the preferences of decision makers (DMs); to the level of DMs' logical thinking, that is, the level of DMs' consistency; consensus is also reflected in the dynamic adjustment nature of the process; and consensus need to aggregate individual opinions in a group decision making (GDM) process.

The first category focuses on the different representation formats of preferences in consensus problems. In GDM processes, different DMs will have and provide opinions in different ways, based on

\footnotetext{
${ }^{*}$ Corresponding author

Email addresses: yguangzhiying@163.com (Ning Zhang), yzwgong26@163.com (Zaiwu Gong), chiclana@dmu.ac.uk (Francisco Chiclana)
} 
their knowledge background, interests, habits and other factors, among others in the form of crisp preferences (Fishburn, 1979), fuzzy preferences (Nurmi, 1981; Fodor and Roubens, 1994; Cabrerizo et al., 2015), interval-valued preferences (Dong et al., 2016), triangular fuzzy preferences (Wu and Chiclana, 2014a), intuitionistic fuzzy numbers (Wu and Chiclana, 2014b; Xu and Liao, 2015), linguistic assessments (Zadeh, 1975; Wu et al., 2015; Cid-Lopez et al., 2017). Moreover, in the selection and evaluation of various alternatives, utility function, preference relation, and preference orderings (Chiclana et al., 1998) are the manifestation of DMs' preference structures. For instance, Chiclana et al. (2001) regarded multiplicative preference relations as a preference representation structure, and constructed a multipurpose decision-making model based on fuzzy preference relations. Jiang et al. (2013) constructed two different consensus models with intuitive multiplicative preference relations, and tested, achieved, and improved the group consensus level. To solve the multi-person decision-making problem with heterogeneous preference representation structures, Zhang et al. (2015) composed a new consensus model, while Dong et al. (2015) constructed a new framework based on the prospect theory and DMs' psychological behaviors. Moreover, Wang and Li (2016) used quadratic programming models to solve GDM with incomplete preference relations. Through a specialized model in the R software environment, Ureña et al. (2016) applied fuzzy preference relations to support fuzzy GDM processes.

The second category deals with individual consistency in consensus problems. It is beneficial to obtain more rational and reliable results by considering individual consistency and group consensus. In this field, $\mathrm{Wu}$ and $\mathrm{Xu}$ (2012) provided a decision support model to promote group consensus while keeping individual consistency for DMs; later, they developed a consensus reaching process for managing individual and group rationality of hesitant fuzzy linguistic preference relations ( $\mathrm{Wu}$ and $\mathrm{Xu}$, 2016). In the process of consensus building with missing values, Wu and Chiclana (2014c) mainly utilized multiplicative consistency of intuitionistic reciprocal preference relations. Driven by the consistency of group decision, Ureña et al. (2015) proposed the method to solve incomplete reciprocal intuitionistic preference relations. In addition, Chiclana et al. (2013) applied different distance functions to individual consistency, and analyzed those different convergence to consensus speeds. Based on a multiplicative analytic hierarchy process (AHP) model with log normal errors, Lin and Kou (2015) put forward a Bayesian revision method for improving the individual pairwise comparison matrices' consistency. Additionally, Li et al. (2017) developed a consistency-driven model based on the two-tuple linguistic model, which benefits reaching consensus with linguistic preference.

The third category addresses dynamic consensus problems. Group consensus itself is a dynamic adjustment process, which reflects active consensus (stigmergy) and non-active consensus. For active consensus within large group emergency decision-making, Xu et al. (2015) presented a dynamic consensus method based on an exit-delegation mechanism, which helped create a solution to satisfy the consistency and consensus criteria simultaneously. By exploring a Markov chain method, Dong and Cooper (2016) structured a peer-to-peer dynamic adaptive consensus reaching model. Moreover, 
Zhao et al. (2017) explored an $H_{\infty}$ sliding mode control model to solve the scaled consensus control problem. For non-active consensus, a moderator of extraordinary leadership participates in a consensus process, which is beneficial to accelerate consensus and improve the efficiency. To minimize the deviation between DMs' and the moderator's opinions, Ben-Arieh and Easton (2007) and Ben-Arieh et al. (2009) adopted the distance-measured method to construct the minimum and minimum quadratic cost consensus models. Generalizing Ben-Arieh's models, Gong et al. (2015a) explored consensus models based on minimum cost and maximum return, and developed consensus models with interval preference opinions. For investigating network-based leader-following consensus problems, He et al. (2017) designed nonlinear multi-agent systems under distributed impulsive control. Dong et al. (2017) exploited a convergent group AHP consensus reaching model with a twofold feedback mechanism, which fully considered the leader or customer opinion in the process of dynamic interaction.

The fourth category relates to information aggregation in consensus problems. To obtain an acceptable consensus result for DMs in GDM, it is feasible to aggregate individual opinion through model construction and graph analysis (Fu and Yang, 2010; Von Winterfeldt and Edwards, 1986). Analytic Hierarchy Process (AHP) (Saaty, 1980) is a systematic and hierarchical analysis method combining qualitative with quantitative information, which is applied to information aggregation problems with significant impact and contribution to group consensus. Indeed, Forman and Peniwati (1998) aggregated DMs' judgements and AHP's priorities to deal with group decision problems. To study the consensus process, Honert (1998) constructed stochastic group preference models based on AHP multiplicative variant. Regan et al. (2006) constructed a mathematical consensus convergent model and demonstrated its utility in an environmental management framework. Besides, Altuzarra et al. (2007) put forward a new priorization procedure based on AHP-group decision making. Srdjevic and Srdjevic (2013) adopted AHP to combine DMs' local priorities, and reduced the group inconsistency; while Srdjevic et al. (2013) designed a two-phase algorithm based on optimal aggregation for consensus. Nevertheless, previous aggregation models focused on crisp, fuzzy, interval, intuitionistic fuzzy, linguistic and other preference representation forms, but rarely referred to opinions/preferences obeying random distributions.

Stochastic programming is a type of mathematical programming that deals with random data, being also an effective tool for modeling optimization problems with random variables. For decades, the stochastic programming problem has been widely used in decision science, investment portfolio, system optimization, and other fields. For instance, Sakawa and Matsui (2013) constructed two-level linear programming problems under fuzzy random environments by considering the cooperative relation between DMs, and derived a satisfactory solution for the upper-level DM. In multi-attribute decision-making problems with interval values and random data, Wu et al. (2016) defined interval number with probability distribution and also identified a new stochastic dominance degree definition. For investment portfolio optimization, Sadati and Nematian (2013) developed a portfolio optimization 
model involving fuzzy random variables, and maximized the possibility and necessity of investment portfolio. By employing random fuzzy variable to describe the stochastic return of individual security with fuzzy information, Qin (2016) exploited mean-absolute deviation portfolio optimization models, while for reducing aircraft noise and optimizing the structural-acoustic system, Wang et al. (2017) proposed a robust optimization method of structural-acoustic coupled systems with random parameters.

Chance constrained programming (Charnes and Cooper, 1959), an important branch of stochastic programming, mainly deals with situations with constraints containing random variables, and the decision must be made before the implementation of these random variables. It is considered that the decision may not satisfy the constraints when the adverse situation occurs, i.e., the decision cannot satisfy the constraint to a certain extent when a principle is adopted, while the probability the constraint should be established is not below a certain confidence level $1-\alpha$. Hong et al. (2011) employed a gradient-based Monte Carlo method to realize convex approximations, which was used for joint chance-constrained programs. To obtain the optimal strategies for carbon capture, utilization, and storage, Dai et al. (2014) exploited an inexact $m_{\lambda}$-measure fuzzy chance-constrained programming. Moreover, for the improvement of wheat crop quality, Bai et al. (2015) instructed a multi-step rolled forward chance-constrained model to deal with uncertain climate in the harvest season. Aiming at the New York center location problem, Gao and Qin (2016) introduced uncertain variables of travel times and established a chance-constrained programming model.

However, opinions are almost always uncertain in GDM, being reflected by interval-valued and intuitionistic fuzzy numbers and other characteristics. Moreover, it is difficult to express DMs' opinions only with probability distribution (e.g., the opinions of DMs can obey a normal distribution with mean $x$ and variance $\sigma^{2}$ ). This article studies the consensus problem when the opinions of DMs or the moderator obey a certain distribution or are intervals. In cost consensus decision making problems (Ben-Arieh and Easton, 2007; Ben-Arieh et al., 2009; Gong et al., 2015a) it is difficult to obtain a cost paid to all DMs for consensus below budget. In this article, it s assumed that the cost paid to all DMs for consensus is lower than the budget under a certain confidence level, i.e., the cost is less than a chosen probability. To obtain the minimum consensus budget under a certain confidence level and to place restrictions on the probability of the cost paid for consensus no more than budget that is not less than a certain confidence level, we construct the cost consensus model based on a random distribution considering the probabilistic budget constraint. The advantages of the new models proposed in the paper are as follows:

- Probability constraints are more realistic than complete constraints;

- Apart from interval values, adding different random distributions can increase the available of uncertain values; 
- With the generalization of the minimum cost consensus models by Ben-Arieh and Easton (2007) and Gong et al. (2015b), our models consider three constraints, including the cost upper limit, the lower DM compensations limit, and the opinion deviation between DMs and the moderator.

The rest of this article is structured as follows. Section 2 introduces the principle and basic assumptions of the stochastic consensus model. Section 3 constructs the cost consensus model based on random distribution, only considering the probabilistic constraint of the moderator's maximum budget. By improving the model, we create a probabilistic constraint of the DM's minimum compensation and the constraint of distance deviation between the moderator's and DM's opinions, and develop a multi-constraint cost consensus model. In Section 4, we use a sensitivity analysis and probabilistic programming based on genetic algorithms (GA), combine them with an application to China's urban demolition negotiation, and simulate the minimum budget in a certain confidence level under different constraints. The article is closed with Section 5 where conclusions are drawn.

\section{Model principle and basic assumptions}

Based on consensus research, by introducing an $\varepsilon$ general consensus concept, Ben-Arieh and Easton (2007) employed a distance-measured method to construct the minimum cost consensus model with the threshold $\varepsilon$ constraint of opinion deviation:

$$
\begin{aligned}
& \operatorname{Min} \varphi=\sum_{i=1}^{m} c_{i}\left|o_{i}^{\prime}-o_{i}\right| \\
& \text { s.t. }\left|o_{i}^{\prime}-o_{i}\right| \leq \varepsilon, i \in M=\{1,2, \ldots, m\} \quad(1-1)
\end{aligned}
$$

where $i \in M=\{1,2, \ldots, m\}$ denotes the $m$ DMs participating in GDM, $o_{i}$ is the original DM $i$ 's opinion, $o_{i}^{\prime}$ denotes $i$ 's opinion after adjustment (consensus opinion), and $\left|o_{i}^{\prime}-o_{i}\right|$ is the deviation between the original opinion $o_{i}$ and the adjusted opinion $o_{i}^{\prime}$. We define $c_{i}$ to be the cost of changing a unit opinion, and the objective function in Model (1) can be interpreted as the minimum cost for obtaining final consensus. In constraint $(1-1), \varepsilon$ is the maximum units of the deviation for reaching common consensus, and so $\left|o_{i}^{\prime}-o_{i}\right| \leq \varepsilon$ denotes the threshold limits of deviation between the DM $i$ 's original and consensus opinion. Subsequently, due to the construction of the minimum quadratic cost consensus model, Ben-Arieh et al. (2009) discussed the problem of single-objective quadratic cost

decision, i.e., changed the objective function to $\sum_{i=1}^{m} c_{i}\left(o_{i}^{\prime}-o_{i}\right)^{2}$, and proposed a linear time algorithm for the minimum consensus cost.

By introducing aggregation operators, Zhang et al. (2013) generalized the minimum cost consensus model, and put forward minimum-cost and maximum expert consensus models. Based on the primal-dual linear programming theory, Gong et al. (2015a) constructed two consensus models based on minimum cost and maximum return regarding the DMs or moderators, and investigated the relationship and economic significance between models and variables.

In realistic decision making, the DMs' opinions often present the following uncertain characteristics: 
- Employing interval values to represent the opinions of DMs and the moderator, and determining the explicit values (i.e., optimal values) of DMs and the moderator using the optimization method, Gong et al. (2015b) constructed the minimum cost consensus model based on interval opinions:

$$
\begin{aligned}
& \operatorname{Min} \varphi=\sum_{i=1}^{m} \omega_{i}\left|o^{\prime}-\left[o_{l i}+\alpha_{i}\left(o_{r i}-o_{l i}\right)\right]\right| \\
& \text { s.t. }\left\{\begin{array}{l}
o^{\prime} \in O \\
o_{i} \in\left[o_{l i}, o_{r i}\right], i \in M \\
0 \leq \alpha_{i} \leq 1, i \in M
\end{array}\right.
\end{aligned}
$$

where $i \in M$ denotes the $m$ DMs participating in GDM, $o_{i}$ is the DM $i$ 's interval valued opinion with lower and upper bounds $o_{l i}$ and $o_{r i}$, respectively. From interval $\left[o_{l i}, o_{r i}\right]$ the following crisp value $o_{l i}+\alpha_{i}\left(o_{r i}-o_{l i}\right)$ is derived, where $0 \leq \alpha_{i} \leq 1$. $o^{\prime}$ is the moderator's opinion, and $O=\left\{o^{\prime} \in \mathbb{R} \mid o^{\prime}>0\right\}$ is the set of all moderators' opinions. To obtain the minimum cost for consensus, the optimal weight $\omega_{i}$ are obtained by Model (2).

- When the opinions of DMs and the moderator present random characteristics, it is difficult to determine the range of opinion by knowing only the opinion distribution. To determine the minimum cost considering random opinions, this paper further generalizes Model (2), and constructs a minimum cost consensus model with random distributed opinions. The basic assumptions of the model are as follows.

Hypothesis 1: The opinions of DMs and the moderator are uncertain, and obey a certain distribution;

Hypothesis 2: Consensus budget is limited. In a budget range, group consensus reaches under a certain confidence level (i.e. a certain probability); in other words, the probability of group consensus is not below the confidence level;

Hypothesis 3: Under the stated certain confidence level, the budget for the reached group consensus has a minimum value.

\section{Model construction}

\subsection{Cost consensus model with single-constraint: cost upper limit}

Assume that $m$ DMs participate in the GDM process, where $o_{i}$ denotes the DM $i(i \in M)$ 's opinion, and $o^{\prime}$ is the moderator's opinion (i.e. $o^{\prime}$ can be interpreted as the group consensus opinion). Here $\omega_{i}$ denotes the unit cost that the moderator is willing to pay DM $i$ to achieve the optimal consensus opinion. Moreover, if $\omega_{i}\left|o^{\prime}-o_{i}\right|$ is used to measure the deviation between the moderator's opinion and DM $i$ 's opinion, then $\omega_{i}\left|o^{\prime}-o_{i}\right|$ is the cost that the moderator pays DM $i$. Therefore, $\sum_{i=1}^{m} \omega_{i}\left|o^{\prime}-o_{i}\right|$ denotes the total cost that the moderator pays all DMs. 
In realistic decision making (Hypothesis 1), the opinions of DMs and the moderator are uncertain, and present a certain distribution. Since these opinions are difficult to be expressed by a certain value, GDM reaches consensus under a certain confidence level $\alpha$ (Hypothesis 2). Next, on the assumption of a certain confidence level, we study the minimum cost for consensus (Hypothesis 3). Let the probability of the cost paid for consensus no more than budget $\beta$ be not less than a certain confidence level $1-\alpha$ ( $\alpha$ is called as significance level): $\operatorname{Pr}\left\{\sum_{i=1}^{m} \omega_{i}\left|o_{i}-o^{\prime}\right| \leq \beta\right\} \geq 1-\alpha$. To measure the minimum budget for the group consensus reached, we construct a consensus model based on the random distribution under the confidence level:

$$
\begin{aligned}
& W(\alpha)=\operatorname{Min} \beta \\
& \text { s.t. } \operatorname{Pr}\left\{\sum_{i=1}^{m} \omega_{i}\left|o_{i}-o^{\prime}\right| \leq \beta\right\} \geq 1-\alpha
\end{aligned}
$$

The following result proves that Model (2) is a special case of Model (3).

Proposition 1. When $\alpha=0$, Model (3) is equivalent to Model (2).

Proof. When $\alpha=0$, Model (3) can be transformed to a general nonlinear programming model:

$$
\begin{aligned}
& W(\alpha)=\operatorname{Min} \beta \\
& \text { s.t. } \sum_{i=1}^{m} \omega_{i}\left|o_{i}-o^{\prime}\right| \leq \beta
\end{aligned}
$$

According to Proposition 1, Model (3) (and in turn Model (4)) generalizes Model (2). The following result proves the monotonicity property of $W(\alpha)$.

Theorem 1. $W(\alpha)$ is a monotonic non-increasing function of significance level $\alpha$.

Proof. Let $\varphi\left(\alpha_{1}\right), \varphi\left(\alpha_{2}\right)$ be the feasible region of Model (3) under significance level $\alpha_{1}$, $\alpha_{2}$. When $\alpha_{1} \leq \alpha_{2}, 1-\alpha_{1} \geq 1-\alpha_{2}$, then $\varphi\left(\alpha_{1}\right) \subseteq \varphi\left(\alpha_{2}\right)$. Thus, $W\left(\alpha_{1}\right) \geq W\left(\alpha_{2}\right)$.

Since the opinions of DM $i$ and the moderator $o^{\prime}$ are uncertain, they are divided into two categories: interval value and probability distribution.

Case 1. DMs' opinions are uncertain, their probability distribution unknown, and the moderator's opinion obeys a certain distribution.

$$
\begin{aligned}
& \operatorname{Min} \beta \\
& \text { s.t. }\left\{\begin{array}{l}
\operatorname{Pr}\left\{\sum_{i=1}^{m} \omega_{i}\left|o_{i}-o^{\prime}\right| \leq \beta\right\} \geq 1-\alpha \\
o_{i} \in\left[o_{i}^{L}, o_{i}^{U}\right], i \in M
\end{array}\right.
\end{aligned}
$$

where the moderator's opinion $o^{\prime}$ obeys a certain distribution, and $o_{i}$ is DM $i$ 's opinion in interval $\left[o_{i}^{L}, o_{i}^{U}\right] . o_{i}^{L}, o_{i}^{U}$ are the lower and upper bounds of DM $i$ 's opinion, respectively. Replacing 
$\left[o_{i}^{L}, o_{i}^{U}\right]$ by its equivalent crisp number $o_{i}^{L}+\mu_{i}\left(o_{i}^{U}-o_{i}^{L}\right)$, Model (5) can be transformed into the following Model (6):

$$
\begin{aligned}
& \operatorname{Min} \beta \\
& \text { s.t. }\left\{\begin{array}{l}
\operatorname{Pr}\left\{\sum_{i=1}^{m} \omega_{i}\left|o_{i}^{L}+\mu_{i}\left(o_{i}^{U}-o_{i}^{L}\right)-o^{\prime}\right| \leq \beta\right\} \geq 1-\alpha \\
0 \leq \mu_{i} \leq 1, i \in M
\end{array}\right.
\end{aligned}
$$

where $o^{\prime}$ obeys a certain distribution.

Case 2. DMs' opinions obey a certain distribution, the moderator's opinion is uncertain and its distribution is unknown.

$$
\begin{aligned}
& \operatorname{Min} \beta \\
& \text { s.t. }\left\{\begin{array}{l}
\operatorname{Pr}\left\{\sum_{i=1}^{m} \omega_{i}\left|o_{i}-o^{\prime}\right| \leq \beta\right\} \geq 1-\alpha \\
o^{\prime} \in\left[o_{L}^{\prime}, o_{U}^{\prime}\right]
\end{array}\right.
\end{aligned}
$$

where DM $i$ 's opinion obeys a certain distribution, and $o^{\prime}$ is the moderator's opinion in interval $\left[o_{L}^{\prime}, o_{U}^{\prime}\right] . o_{L}^{\prime}, o_{U}^{\prime}$ are the lower and upper bounds of the moderator's opinion respectively. Replacing $\left[o_{L}^{\prime}, o_{U}^{\prime}\right]$ by its equivalent crisp number $o_{L}^{\prime}+\mu\left(o_{U}^{\prime}-o_{L}^{\prime}\right)$, Model (7) can be transformed into the following Model (8):

$$
\begin{aligned}
& \text { Min } \beta \\
& \text { s.t. }\left\{\begin{array}{l}
\operatorname{Pr}\left\{\sum_{i=1}^{m} \omega_{i}\left|o_{i}-\left[o_{L}^{\prime}+\mu\left(o_{U}^{\prime}-o_{L}^{\prime}\right)\right]\right| \leq \beta\right\} \geq 1-\alpha \\
0 \leq \mu \leq 1
\end{array}\right.
\end{aligned}
$$

where $o_{i}, i \in M$ obeys a certain distribution.

The significance of the objective function in Models (5)-(8) is that whatever $\beta$ is, consensus can be realized/reached with confidence level of $1-\alpha$.

\subsection{Cost consensus model with two-constraints: upper cost limit and lower DM compensation limit}

From the moderator point of view, the lower the group consensus budget the better, as he/she aims at the budget to not exceed a certain upper limit. However, for the DMs, it is unfavorable to reach group consensus with a low budget, and they hope the compensation is as large as possible because they sacrifice individual interests for the benefit of the group. Similarly, from the perspective of the moderator, the probability of the cost paid for consensus being no more than budget $\beta$ is set to be not less than a certain confidence level $1-\alpha_{1}$, i.e. $\operatorname{Pr}\left\{\sum_{i=1}^{m} \omega_{i}\left|o_{i}-o^{\prime}\right| \leq \beta\right\} \geq 1-\alpha_{1}$. However, the DMs must be able to obtain compensation for reaching consensus, so let the cost paid for consensus be used to guarantee the lowest reward $\gamma$, and the probability of exceeding the lowest reward $\gamma$ be not less than another confidence level $1-\alpha_{2}$, i.e. $\operatorname{Pr}\left\{\sum_{i=1}^{m} \omega_{i}\left|o_{i}-o^{\prime}\right| \geq \gamma\right\} \geq 1-\alpha_{2}$. Under the constraints 
of the above two conditions, to discuss the minimum of $\beta-\gamma$, we construct the following Model (9):

$$
\begin{aligned}
& \operatorname{Min} \beta-\gamma \\
& \text { s.t. }\left\{\begin{array}{l}
\operatorname{Pr}\left\{\sum_{i=1}^{m} \omega_{i}\left|o_{i}-o^{\prime}\right| \leq \beta\right\} \geq 1-\alpha_{1} \\
\operatorname{Pr}\left\{\sum_{i=1}^{m} \omega_{i}\left|o_{i}-o^{\prime}\right| \geq \gamma\right\} \geq 1-\alpha_{2}
\end{array}\right.
\end{aligned}
$$

As before, two cases are possible:

Case 1. DMs' opinions are uncertain wth unknown probability distribution, and the moderator's opinion obeys a certain distribution.

$$
\begin{aligned}
& \operatorname{Min} \beta-\gamma \\
& \text { s.t. }\left\{\begin{array}{l}
\operatorname{Pr}\left\{\sum_{i=1}^{m} \omega_{i}\left|o_{i}-o^{\prime}\right| \leq \beta\right\} \geq 1-\alpha_{1} \\
\operatorname{Pr}\left\{\sum_{i=1}^{m} \omega_{i}\left|o_{i}-o^{\prime}\right| \geq \gamma\right\} \geq 1-\alpha_{2} \\
o_{i} \in\left[o_{i}^{L}, o_{i}^{U}\right], i \in M
\end{array}\right.
\end{aligned}
$$

Model (10) can be transformed into the following Model (11):

$$
\begin{aligned}
& \operatorname{Min} \beta-\gamma \\
& \text { s.t. }\left\{\begin{array}{l}
\operatorname{Pr}\left\{\sum_{i=1}^{m} \omega_{i}\left|o_{i}^{L}+\mu_{i}\left(o_{i}^{U}-o_{i}^{L}\right)-o^{\prime}\right| \leq \beta\right\} \geq 1-\alpha_{1} \\
\operatorname{Pr}\left\{\sum_{i=1}^{m} \omega_{i}\left|o_{i}^{L}+\mu_{i}\left(o_{i}^{U}-o_{i}^{L}\right)-o^{\prime}\right| \geq \gamma\right\} \geq 1-\alpha_{2} \\
0 \leq \mu_{i} \leq 1, i \in M
\end{array}\right.
\end{aligned}
$$

Case 2. DMs' opinions obey a certain distribution, the moderator's opinion is uncertain and its distribution is unknown.

$$
\begin{aligned}
& \operatorname{Min} \beta-\gamma \\
& \text { s.t. }\left\{\begin{array}{l}
\operatorname{Pr}\left\{\sum_{i=1}^{m} \omega_{i}\left|o_{i}-o^{\prime}\right| \leq \beta\right\} \geq 1-\alpha_{1} \\
\operatorname{Pr}\left\{\sum_{i=1}^{m} \omega_{i}\left|o_{i}-o^{\prime}\right| \geq \gamma\right\} \geq 1-\alpha_{2} \\
o^{\prime} \in\left[o_{L}^{\prime}, o_{U}^{\prime}\right]
\end{array}\right.
\end{aligned}
$$

Model (12) can be transformed into the following Model (13):

$$
\begin{aligned}
& \operatorname{Min} \beta-\gamma \\
& \text { s.t. }\left\{\begin{array}{l}
\operatorname{Pr}\left\{\sum_{i=1}^{m} \omega_{i}\left|o_{i}-\left[o_{L}^{\prime}+\mu\left(o_{U}^{\prime}-o_{L}^{\prime}\right)\right]\right| \leq \beta\right\} \geq 1-\alpha_{1} \\
\operatorname{Pr}\left\{\sum_{i=1}^{m} \omega_{i}\left|o_{i}-\left[o_{L}^{\prime}+\mu\left(o_{U}^{\prime}-o_{L}^{\prime}\right)\right]\right| \geq \gamma\right\} \geq 1-\alpha_{2} \\
0 \leq \mu \leq 1
\end{array}\right.
\end{aligned}
$$

Thus, we can similarly discuss the minimum of $\beta-\gamma$, and the change in $\beta-\gamma$ with the change in $\alpha$. 
3.3. Cost consensus model with three-constraints: cost upper limit, lower DM compensations limit, and opinion deviation between DMs and the moderator

In GDM, the role of the moderator is similar to a leader's role in an organization, where he/she has good communication and negotiation skills, and leads the development trend of the entire group decision to approach the moderator's opinion. In some cases, the moderator needs to guide (control) some DMs, and to guarantee that the opinion deviation between these DMs and himself/herself is not too large, i.e. the opinions deviation is within a certain range $\left(\left|o_{s}-o^{\prime}\right| \leq \zeta, s \in S \subseteq M\right)$. Based on Subsection 3.2., we construct the following consensus model (Model (14)) based on the random distribution subject to the above opinion constraint:

$$
\begin{aligned}
& \operatorname{Min} \beta-\gamma \\
& \text { s.t. }\left\{\begin{array}{l}
\operatorname{Pr}\left\{\sum_{i=1}^{m} \omega_{i}\left|o_{i}-o^{\prime}\right| \leq \beta\right\} \geq 1-\alpha_{1} \\
\operatorname{Pr}\left\{\sum_{i=1}^{m} \omega_{i}\left|o_{i}-o^{\prime}\right| \geq \gamma\right\} \geq 1-\alpha_{2} \\
\left|o_{s}-o^{\prime}\right| \leq \zeta, s \in S \subseteq M
\end{array}\right.
\end{aligned}
$$

Again, two cases are possible:

Case 1. DMs' opinions are uncertain, probability distribution is unknown, and the moderator's opinion obeys a certain distribution.

$$
\begin{aligned}
& \operatorname{Min} \beta-\gamma \\
& \text { s.t. }\left\{\begin{array}{l}
\operatorname{Pr}\left\{\sum_{i=1}^{m} \omega_{i}\left|o_{i}-o^{\prime}\right| \leq \beta\right\} \geq 1-\alpha_{1} \\
\operatorname{Pr}\left\{\sum_{i=1}^{m} \omega_{i}\left|o_{i}-o^{\prime}\right| \geq \gamma\right\} \geq 1-\alpha_{2} \\
\left|o_{s}-o^{\prime}\right| \leq \zeta, s \in S \subseteq M \\
o_{i} \in\left[o_{i}^{L}, o_{i}^{U}\right], i \in M
\end{array}\right.
\end{aligned}
$$

Model (15) can be transformed into Model (16):

$$
\begin{aligned}
& \operatorname{Min} \beta-\gamma \\
& \text { s.t. }\left\{\begin{array}{l}
\operatorname{Pr}\left\{\sum_{i=1}^{m} \omega_{i}\left|o_{i}^{L}+\mu_{i}\left(o_{i}^{U}-o_{i}^{L}\right)-o^{\prime}\right| \leq \beta\right\} \geq 1-\alpha_{1} \\
\operatorname{Pr}\left\{\sum_{i=1}^{m} \omega_{i}\left|o_{i}^{L}+\mu_{i}\left(o_{i}^{U}-o_{i}^{L}\right)-o^{\prime}\right| \geq \gamma\right\} \geq 1-\alpha_{2} \\
\left|o_{s}-o^{\prime}\right| \leq \zeta, s \in S \subseteq M \\
0 \leq \mu_{i} \leq 1, i \in M
\end{array}\right.
\end{aligned}
$$

Case 2. DMs' opinions obey a certain distribution, the moderator's opinion is uncertain and its 
distribution is unknown.

$$
\begin{aligned}
& \operatorname{Min} \beta-\gamma \\
& \text { s.t. }\left\{\begin{array}{l}
\operatorname{Pr}\left\{\sum_{i=1}^{m} \omega_{i}\left|o_{i}-o^{\prime}\right| \leq \beta\right\} \geq 1-\alpha_{1} \\
\operatorname{Pr}\left\{\sum_{i=1}^{m} \omega_{i}\left|o_{i}-o^{\prime}\right| \geq \gamma\right\} \geq 1-\alpha_{2} \\
\left|o_{s}-o^{\prime}\right| \leq \zeta, s \in S \subseteq M \\
o^{\prime} \in\left[o_{L}^{\prime}, o_{U}^{\prime}\right]
\end{array}\right.
\end{aligned}
$$

Model (17) can be transformed into Model (18):

$$
\begin{aligned}
& \operatorname{Min} \beta-\gamma \\
& \text { s.t. }\left\{\begin{array}{l}
\operatorname{Pr}\left\{\sum_{i=1}^{m} \omega_{i}\left|o_{i}-\left[o_{L}^{\prime}+\mu\left(o_{U}^{\prime}-o_{L}^{\prime}\right)\right]\right| \leq \beta\right\} \geq 1-\alpha_{1} \\
\operatorname{Pr}\left\{\sum_{i=1}^{m} \omega_{i}\left|o_{i}-\left[o_{L}^{\prime}+\mu\left(o_{U}^{\prime}-o_{L}^{\prime}\right)\right]\right| \geq \gamma\right\} \geq 1-\alpha_{2} \\
\left|o_{s}-o^{\prime}\right| \leq \zeta, s \in S \subseteq M \\
0 \leq \mu \leq 1
\end{array}\right.
\end{aligned}
$$

Again, we can discuss the minimum of $\beta-\gamma$, and the change in $\beta-\gamma$ with the change in $\alpha$.

\section{Application to the minimum cost consensus models based on China's urban demolition negotiation}

In urban relocation negotiations, the government plays the role of moderator while urban residents are DMs. Naturally, the government needs to negotiate with residents regarding the compensation price. Consensus is reached only when all residents' expected and the government's compensation price reach an agreement, i.e. the expected utilities of all residents' expected compensation are largely satisfied. Taking the background of China's urban demolition negotiation (Gong et al., 2017), we assume the demolition negotiation involves four residents $d_{1}, d_{2}, d_{3}, d_{4}$, and their expected compensation price intervals are $o_{1} \in[48,52], o_{2} \in[50,55], o_{3} \in[60,65]$, and $o_{4} \in[62,67]$. Meanwhile, the government's compensation price is $o^{\prime} \in[50,65]$ (unit: ten thousands RMB). To reach the final relocation consensus, the unit negotiation costs that the government pays residents are $\omega_{1}=0.8$, $\omega_{2}=1.5, \omega_{3}=1.2$, and $\omega_{4}=0.9$ (unit: thousand RMB). In the following, we discuss situations for demolition consensus considering interval-valued and random prices.

\subsection{Cost consensus model with single-constraint: cost upper limit}

Case 1. $o_{i} \in\left[o_{i}^{L}, o_{i}^{U}\right]$ with unknown distribution, and $o^{\prime}$ obeys a certain distribution. According to the $3 \sigma$ principle of normally distributed variables (Chen et al., 2016), it is known that a random normally distributed variable $X \sim N\left(\mu, \sigma^{2}\right)$ verifies $P(\mu-3 \sigma \leq X \leq \mu+3 \sigma) \approx 1$. Thus, interval values can be linked to a random normally distributed variable using the relation

$$
\left[a^{-}, a^{+}\right]=[\mu-3 \sigma, \mu+3 \sigma], \mu=\frac{a^{-}+a^{+}}{2}, \sigma=\frac{a^{+}-a^{-}}{6}
$$


Using (19), we obtain the distribution of the government's compensation price $o^{\prime} \sim N\left(57.5,2.5^{2}\right)$, i.e. the government's expected compensation price is 57.5 with variance $2.5^{2}$. Setting the probability of the cost paid for consensus no more than the budget not less than 0.9 , the optimization consensus model (Model (6)) based on the minimum cost becomes:

$$
\begin{aligned}
& \operatorname{Min} \beta \\
& \text { s.t. }\left\{\begin{array}{l}
\operatorname{Pr}\left\{0.8 *\left|\left(48+4 \mu_{1}\right)-o^{\prime}\right|+1.5 *\left|\left(50+5 \mu_{2}\right)-o^{\prime}\right|+\right. \\
\left.1.2 *\left|\left(60+5 \mu_{3}\right)-o^{\prime}\right|+0.9 *\left|\left(62+5 \mu_{4}\right)-o^{\prime}\right| \leq \beta\right\} \geq 0.9 \\
o^{\prime} \sim N\left(57.5,2.5^{2}\right) \\
0 \leq \mu_{i} \leq 1, i=1,2,3,4
\end{array}\right.
\end{aligned}
$$

Case 2. $o_{i}$ obeys a certain distribution; $o^{\prime} \in\left[o_{L}^{\prime}, o_{U}^{\prime}\right]$ with unknown distribution. Applying again (19), the four residents' expected compensation price distributions are $o_{1} \sim N\left(50,0.6667^{2}\right), o_{2} \sim$ $N\left(52.5,0.8333^{2}\right), o_{3} \sim N\left(62.5,0.8333^{2}\right)$, and $o_{4} \sim N\left(64.5,0.8333^{2}\right)$. Similarly, the optimization consensus model (Model (8)) based on the minimum cost becomes:

$$
\begin{aligned}
& \operatorname{Min} \beta \\
& \text { s.t. }\left\{\begin{array}{l}
\operatorname{Pr}\left\{0.8 *\left|(50+15 \mu)-o_{1}\right|+1.5 *\left|(50+15 \mu)-o_{2}\right|+\right. \\
\left.1.2 *\left|(50+15 \mu)-o_{3}\right|+0.9 *\left|(50+15 \mu)-o_{4}\right| \leq \beta\right\} \geq 0.9 \\
o_{1} \sim N\left(50,0.6667^{2}\right) \\
o_{2} \sim N\left(52.5,0.8333^{2}\right) \\
o_{3} \sim N\left(62.5,0.8333^{2}\right) \\
o_{4} \sim N\left(64.5,0.8333^{2}\right) \\
0 \leq \mu \leq 1
\end{array}\right.
\end{aligned}
$$

The solutions of these optimization consensus models are shown in Table 1 . The final relocation consensus has a probability of $90 \%=1-10 \%$ with the conditions of Case 1 (when Min $\beta=19.0451$ ) and Case 2 (when $\operatorname{Min} \beta=27.1073$ ).

Table 1: Results of the cost consensus models with single-constraint

\begin{tabular}{ccccccc}
\hline & $o_{1}$ & $o_{2}$ & $o_{3}$ & $o_{4}$ & $o^{\prime}$ & $\beta$ \\
\hline Case 1 & 51.9664 & 54.3240 & 61.4760 & 62.1320 & - & 19.0451 \\
\hline Case 2 & - & - & - & - & 53.2295 & 27.1073 \\
\hline
\end{tabular}

Moreover to discuss the influence of the budget with different distributions, we consider the normal distribution, and the uniform distribution. The results are shown in Fig. 1. Case 1 is represented in the top figure: on the $\mathrm{x}$-axis 1 represents the moderator's opinion obeys the normal distribution, 
while 2 represents the moderator's opinion obeys the uniform distribution. Case 2 is represented in the botton figure: 1 on the $\mathrm{x}$-axis 1 represents the DMs' opinions obey the normal distribution, while 2 represents the DMs' opinions obey the uniform distribution.
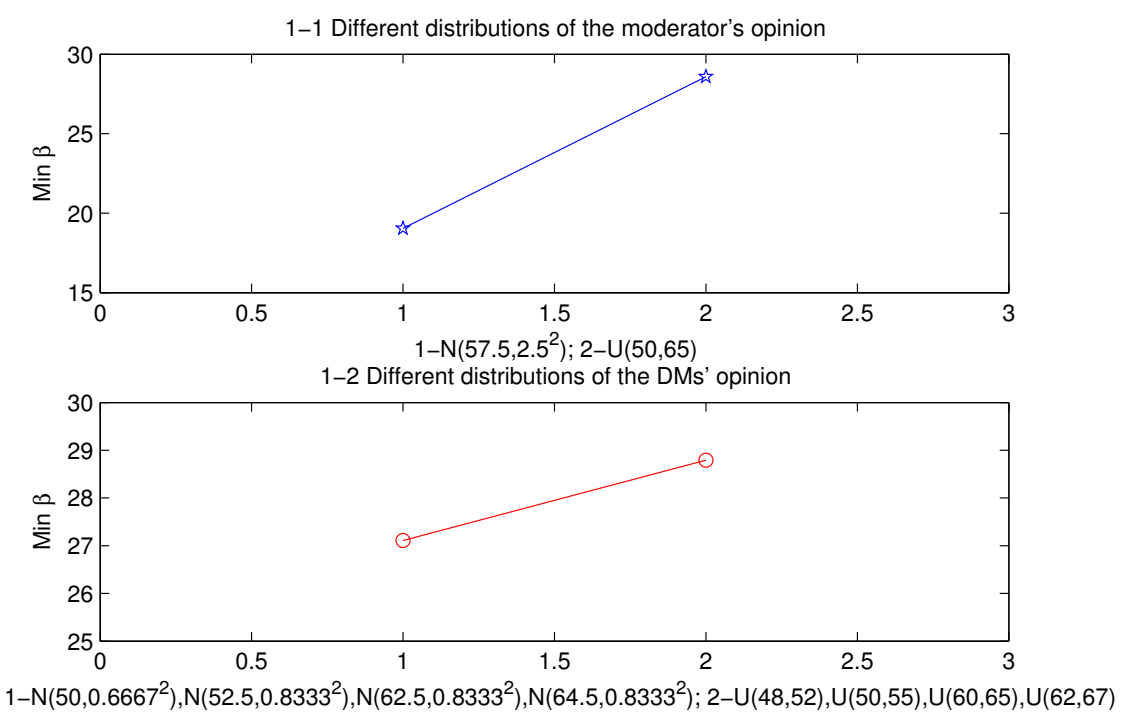

Figure 1: Different opinion distributions with single-constraint.

Taking Case 1 as an example, the change in budget $\beta$ is shown in Fig. 2 with the change in the confidence level, $1-\alpha$, and the distributions of the moderator's opinion. The confidence level $1-\alpha$ values shown are $1,0.9,0.8,0.7,0.6$, and 0.5 ; and the distributions the moderator's opinion being $N\left(57.5,2.5^{2}\right)$ and $U(50,65)$. It is clear that the minimum budget decreases as the significance level $\alpha$ increases, i.e. the confidence level decreases.

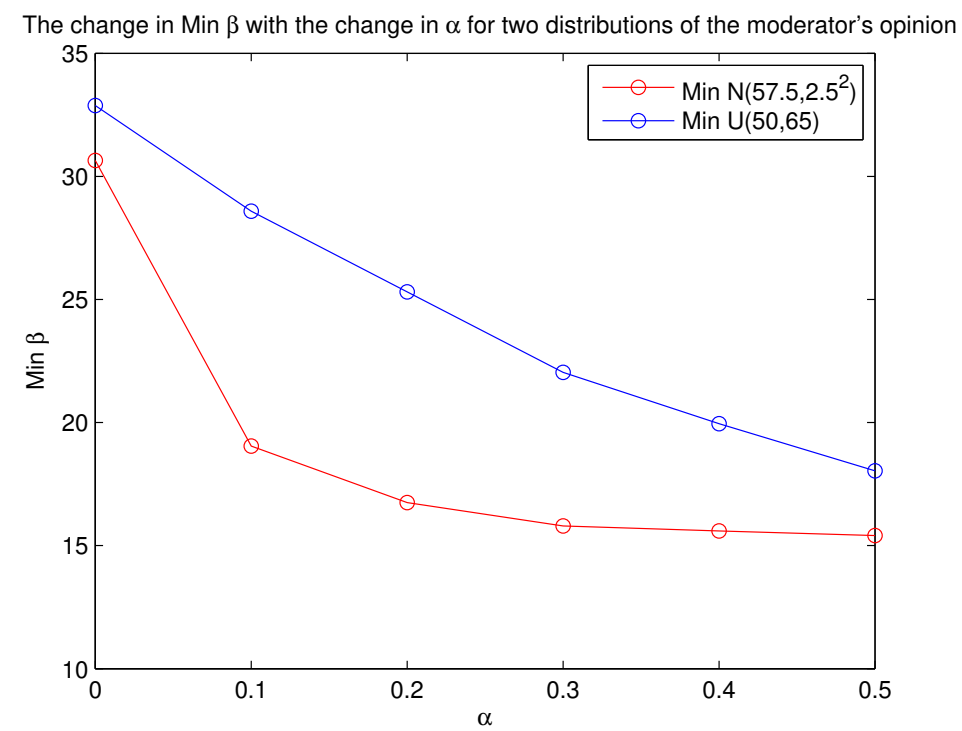

Figure 2: The change in $\operatorname{Min} \beta$ with the change in $\alpha$ for three distributions of the moderator's opinion 
4.2. The cost consensus model with two-constraints: the upper limit of the cost and the lower limit of DMs' compensations

Case 1. $o_{i} \in\left[o_{i}^{L}, o_{i}^{U}\right]$, the distribution of $o_{i}$ is unknown; and $o^{\prime}$ obeys a certain distribution. In urban relocation negotiations, we assume the conditions are the same as in Case 1 in Section 4.1. We not only let the probability of the cost paid for consensus no more than budget be not less than 0.9 , but also let the probability of the cost paid for consensus exceeding the lowest reward be not less than 0.9. The optimization consensus model (Model (11)) based on the minimum cost is:

$$
\begin{aligned}
& \text { Min } \beta-\gamma \\
& \text { s.t. }\left\{\begin{array}{l}
\operatorname{Pr}\left\{0.8 *\left|\left(48+4 \mu_{1}\right)-o^{\prime}\right|+1.5 *\left|\left(50+5 \mu_{2}\right)-o^{\prime}\right|+\right. \\
\left.1.2 *\left|\left(60+5 \mu_{3}\right)-o^{\prime}\right|+0.9 *\left|\left(62+5 \mu_{4}\right)-o^{\prime}\right| \leq \beta\right\} \geq 0.9 \\
\operatorname{Pr}\left\{0.8 *\left|\left(48+4 \mu_{1}\right)-o^{\prime}\right|+1.5 *\left|\left(50+5 \mu_{2}\right)-o^{\prime}\right|+\right. \\
\left.1.2 *\left|\left(60+5 \mu_{3}\right)-o^{\prime}\right|+0.9 *\left|\left(62+5 \mu_{4}\right)-o^{\prime}\right| \geq \gamma\right\} \geq 0.9 \\
o^{\prime} \sim N\left(57.5,2.5^{2}\right) \\
0 \leq \mu_{i} \leq 1, i=1,2,3,4
\end{array}\right.
\end{aligned}
$$

Case 2. $o_{i}$ obeys a certain distribution; $o^{\prime} \in\left[o_{L}^{\prime}, o_{U}^{\prime}\right]$, and the distribution of $o^{\prime}$ is unknown. We assume the conditions are the same as in Case 2 in Section 4.1. We not only let the probability of the cost paid for consensus no more than budget be not less than 0.9 , but also let the probability of the cost paid for consensus exceeding the lowest reward be not less than 0.9. The optimization consensus model (Model (13)) based on the minimum cost is:

$$
\begin{aligned}
& \text { Min } \beta-\gamma \\
& \text { s.t. }\left\{\begin{array}{l}
\operatorname{Pr}\left\{0.8 *\left|(50+15 \mu)-o_{1}\right|+1.5 *\left|(50+15 \mu)-o_{2}\right|+\right. \\
\left.1.2 *\left|(50+15 \mu)-o_{3}\right|+0.9 *\left|(50+15 \mu)-o_{4}\right| \leq \beta\right\} \geq 0.9 \\
\operatorname{Pr}\left\{0.8 *\left|(50+15 \mu)-o_{1}\right|+1.5 *\left|(50+15 \mu)-o_{2}\right|+\right. \\
\left.1.2 *\left|(50+15 \mu)-o_{3}\right|+0.9 *\left|(50+15 \mu)-o_{4}\right| \geq \gamma\right\} \geq 0.9 \\
o_{1} \sim N\left(50,0.6667^{2}\right) \\
o_{2} \sim N\left(52.5,0.8333^{2}\right) \\
o_{3} \sim N\left(62.5,0.8333^{2}\right) \\
o_{4} \sim N\left(64.5,0.8333^{2}\right) \\
0 \leq \mu \leq 1
\end{array}\right.
\end{aligned}
$$

The solutions of these optimization consensus models are shown in Table 2. The final relocation consensus has a probability of $90 \%=1-10 \%$ with the conditions of Case 1 (when Min $\beta-\gamma=1.2002$ ) and Case 2 (when Min $\beta-\gamma=3.6950$ ). Similarly, Fig. 3 shows results that help to discuss the influence of the minimum budget with different distributions, and it is to read similarly as Fig. 1 above. 
Table 2: Results of the cost consensus models with two-constraints

\begin{tabular}{ccccccc}
\hline & $o_{1}$ & $o_{2}$ & $o_{3}$ & $o_{4}$ & $o^{\prime}$ & $\beta-\gamma$ \\
\hline Case 1 & 51.9972 & 52.9920 & 64.3750 & 66.1140 & - & 1.2002 \\
\hline Case 2 & - & - & - & - & 52.4690 & 3.6950 \\
\hline
\end{tabular}
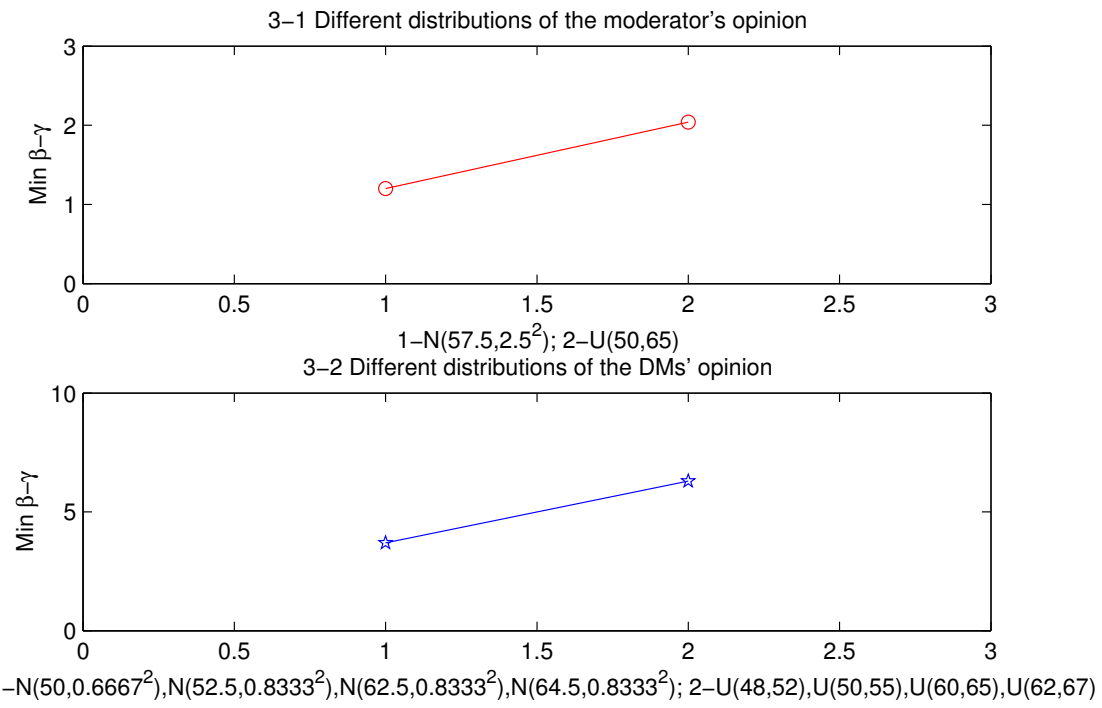

Figure 3: Different opinion distributions with two-constraints

4.3. Cost consensus model with three-constraints: cost upper limit, lower DM compensations limit, and opinion deviation between DMs and the moderator

Case 1. $o_{i} \in\left[o_{i}^{L}, o_{i}^{U}\right]$ with unknown distribution, and $o^{\prime}$ obeys a certain distribution. In urban relocation negotiations, we assume the same conditions as in Case 1 in Subsection 4.1. We add the deviation constraints between four residents' expected compensation prices and the government's compensation price, and the optimization consensus model (Model (16)) based on the minimum cost is:

$$
\begin{aligned}
& \operatorname{Min} \beta-\gamma \\
& \text { s.t. }\left\{\begin{array}{l}
\operatorname{Pr}\left\{0.8 *\left|\left(48+4 \mu_{1}\right)-o^{\prime}\right|+1.5 *\left|\left(50+5 \mu_{2}\right)-o^{\prime}\right|+\right. \\
\left.1.2 *\left|\left(60+5 \mu_{3}\right)-o^{\prime}\right|+0.9 *\left|\left(62+5 \mu_{4}\right)-o^{\prime}\right| \leq \beta\right\} \geq 0.9 \\
\operatorname{Pr}\left\{0.8 *\left|\left(48+4 \mu_{1}\right)-o^{\prime}\right|+1.5 *\left|\left(50+5 \mu_{2}\right)-o^{\prime}\right|+\right. \\
\left.1.2 *\left|\left(60+5 \mu_{3}\right)-o^{\prime}\right|+0.9 *\left|\left(62+5 \mu_{4}\right)-o^{\prime}\right| \geq \gamma\right\} \geq 0.9 \\
\operatorname{Min}\left\{\left|48+4 \mu_{1}-o^{\prime}\right|,\left|50+5 \mu_{2}-o^{\prime}\right|,\right. \\
\left.\left|60+5 \mu_{3}-o^{\prime}\right|,\left|62+5 \mu_{4}-o^{\prime}\right|\right\} \leq 10 \\
o^{\prime} \sim N\left(57.5,2.5^{2}\right) \\
0 \leq \mu_{i} \leq 1, i=1,2,3,4
\end{array}\right.
\end{aligned}
$$

Case 2. $o_{i}$ obeys a certain distribution; $o^{\prime} \in\left[o_{L}^{\prime}, o_{U}^{\prime}\right]$ with unknown distribution. With the same conditions of Case 2 in Subsection 4.1, and the addition of the deviation constraints between 
four residents' expected compensation prices and the government's compensation price, the optimization consensus model (Model (18)) based on the minimum cost is:

$$
\begin{aligned}
& \operatorname{Min} \beta-\gamma \\
& \text { s.t. }\left\{\begin{array}{l}
\operatorname{Pr}\left\{0.8 *\left|(50+15 \mu)-o_{1}\right|+1.5 *\left|(50+15 \mu)-o_{2}\right|+\right. \\
\left.1.2 *\left|(50+15 \mu)-o_{3}\right|+0.9 *\left|(50+15 \mu)-o_{4}\right| \leq \beta\right\} \geq 0.9 \\
\operatorname{Pr}\left\{0.8 *\left|(50+15 \mu)-o_{1}\right|+1.5 *\left|(50+15 \mu)-o_{2}\right|+\right. \\
\left.1.2 *\left|(50+15 \mu)-o_{3}\right|+0.9 *\left|(50+15 \mu)-o_{4}\right| \geq \gamma\right\} \geq 0.9 \\
\operatorname{Min}\left\{\left|(50+15 \mu)-o_{1}\right|, \mid 50+15 \mu\right)-o_{2} \mid, \\
\left.\left|(50+15 \mu)-o_{3}\right|,\left|(50+15 \mu)-o_{4}\right|\right\} \leq 15 \\
o_{1} \sim N\left(50,0.6667^{2}\right) \\
o_{2} \sim N\left(52.5,0.8333^{2}\right) \\
o_{3} \sim N\left(62.5,0.8333^{2}\right) \\
o_{4} \sim N\left(64.5,0.8333^{2}\right) \\
0 \leq \mu \leq 1
\end{array}\right.
\end{aligned}
$$

The solutions of these optimization consensus models are shown in Table 3. The final relocation consensus has a probability of $90 \%=1-10 \%$ with the conditions of Case 1 (when $\operatorname{Min} \beta-\gamma=1.5111$ ) and Case 2 (when Min $\beta-\gamma=3.6978$ ). Similarly, Fig. 4 helps in discussing the influence of the minimum budget with different distributions.

Table 3: Results of the cost consensus models with three-constraints

\begin{tabular}{ccccccc}
\hline & $o_{1}$ & $o_{2}$ & $o_{3}$ & $o_{4}$ & $o^{\prime}$ & $\beta-\gamma$ \\
\hline Case 1 & 50.9672 & 52.3935 & 64.9995 & 65.7540 & - & 1.5111 \\
\hline Case 2 & - & - & - & - & 52.4420 & 3.6978 \\
\hline
\end{tabular}

\subsection{The effectiveness of the proposed probabilistic programming method based on GA}

To demonstrate the effectiveness of the proposed probabilistic programming method based on genetic algorithms (GA) (details in Appendix I), we compute Gong et al. (2017) model by our algorithm as shown below in (20), with the iteration results shown in Fig. 5.

$$
\begin{aligned}
& \text { Min } 0.8 *\left|\left(48+4 \mu_{1}\right)-\left(50+15 \mu_{5}\right)\right|+1.5 *\left|\left(50+5 \mu_{2}\right)-\left(50+15 \mu_{5}\right)\right|+ \\
& 1.2 *\left|\left(60+5 \mu_{3}\right)-\left(50+15 \mu_{5}\right)\right|+0.9 *\left|\left(62+5 \mu_{4}\right)-\left(50+15 \mu_{5}\right)\right| \\
& \text { s.t. } 0 \leq \mu_{i} \leq 1, i=1,2,3,4,5
\end{aligned}
$$

Compared with the optimal minimum negotiation budget of 14.7 obtained in (Gong et al., 2017), it is clear that our iteration results produce an optimal result close to the above one after 200 iterations. Apart from interval values, our algorithm can also deal with fuzzy numbers obeyed random 

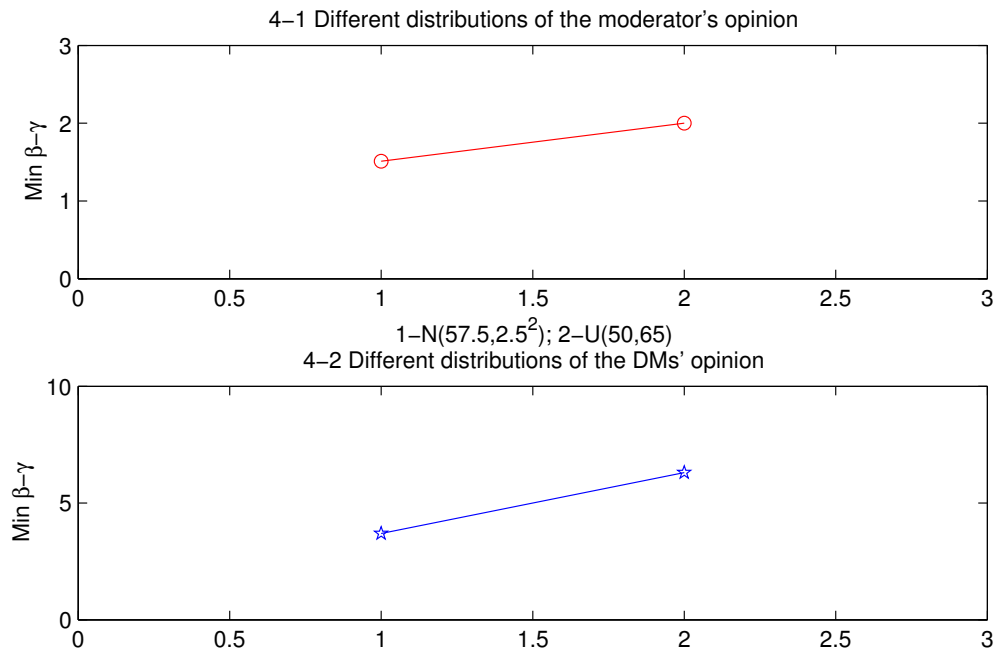

$1-\mathrm{N}\left(50,0.6667^{2}\right), \mathrm{N}\left(52.5,0.8333^{2}\right), \mathrm{N}\left(62.5,0.8333^{2}\right), \mathrm{N}\left(64.5,0.8333^{2}\right) ; 2-\mathrm{U}(48,52), \mathrm{U}(50,55), \mathrm{U}(60,65), \mathrm{U}(62,67)$

Figure 4: Different opinion distributions with three-constraints

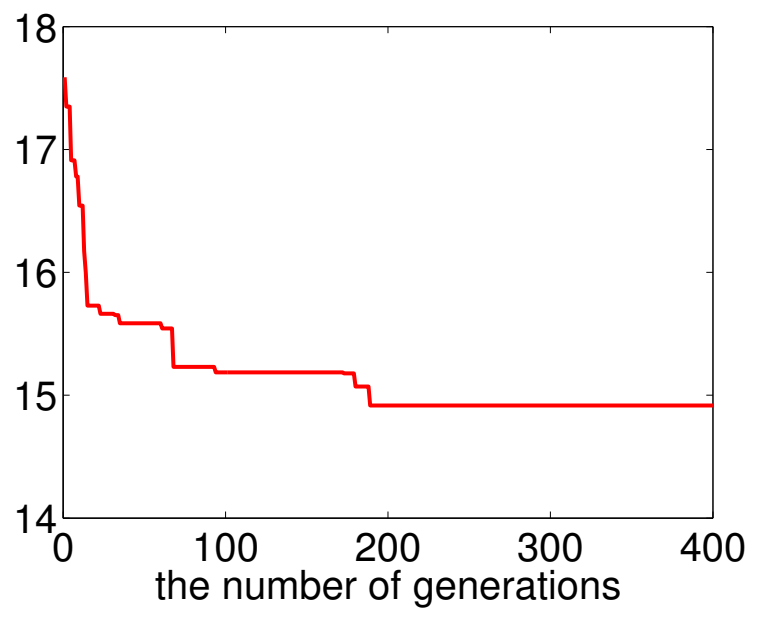

Figure 5: The iteration results of Gong's model computed with proposed algorithm 
distributions. The detail steps of the proposed probabilistic programming method based on GA are as follows:

Step 1: randomly generate initial population between all variables' stated ranges based on GA;

Step 2,: randomly generate some variables obeyed a certain distribution;

Step 3: combined initial population with random variables, evaluate whether all generated variables satisfy the constraints. If yes, then go Step 5; if no, then go Step 4;

Step 4: through selective reproduction, crossing over and mutation, go into generation cycle and go Step 2;

Step 5: collect all feasible populations and obtain the optimal solution.

\section{Conclusions}

Uncertain opinions often exist in GDM and obstruct the consensus process. Except for interval values, we assume that the opinions of DMs and the moderator obey a certain distribution, and construct the cost consensus model based on random distribution with a probability of budget constraints. The objective function of the stochastic optimization model is to obtain the minimum of the total budget under a certain confidence level. The constraints of the model include: the probability of the cost paid for consensus no more than the budget is not less than a certain confidence level; the probability of the cost paid for consensus, which exceeds the lowest reward, is not less than another confidence level; and the qualified opinion deviations between the moderator and some DMs do not exceed a certain threshold. Additionally, for the China's urban demolition negotiation application, we design the minimum cost consensus models and introduce GA to solve the corresponding stochastic optimization models. The algorithm not only has better convergence, but also can better simulate the consensus GDM process, providing a satisfactory solution to the stochastic optimization consensus model.

The main innovative contributions of this article can be summarized as follows:

- The cost consensus model based on random opinion distribution is not only the generalization of the minimum cost consensus model by Ben-Arieh and Easton (2007), but also the further improvement of the consensus model with uncertain opinions by Gong et al. (2015b).

- The opinions of DMs and the moderator cab be assumed to obey different distributions, such as normal distribution, uniform distribution, Chi-square distribution, etc, which improves the expression forms of uncertain opinions and are more relevant to reality.

- Probabilistic planning based on GA is used to resolve the model, which has the advantages of good convergence, high precision, and saving computation time. 
- In the case analysis, the sensitivity analysis method is adopted to obtain the lower bounds of the budget, and the minimum budget is obtained under different constraints or various opinions distributions.

\section{Appendix I}

Probabilistic programming (Kushmerick et al., 1995) is used to deal with goal programming problems with probabilistic constraints. In this article, we explore a new probabilistic programming method based on GA, which combines probabilistic programming with goal programming problems. GA is a random search method referenced in biological evolution with regularity (survival of the fittest) and first proposed by Holland (1992). Its main characteristics are as follows: it operates directly on the structure objects, and there is no limit of derivation and function continuity; it has conceal concurrence and splendid capability with the better situation as a whole; it uses probability optimization methods to automatically obtain and optimize the search space, adaptively adjust the search direction, but does not need to determine the rules. With these properties, GA has been widely used in combinatorial optimization, machine learning, signal processing, adaptive control, and artificial life. It is a key technique in modern intelligent computing (the basic operation of GA is shown in Fig. A1).

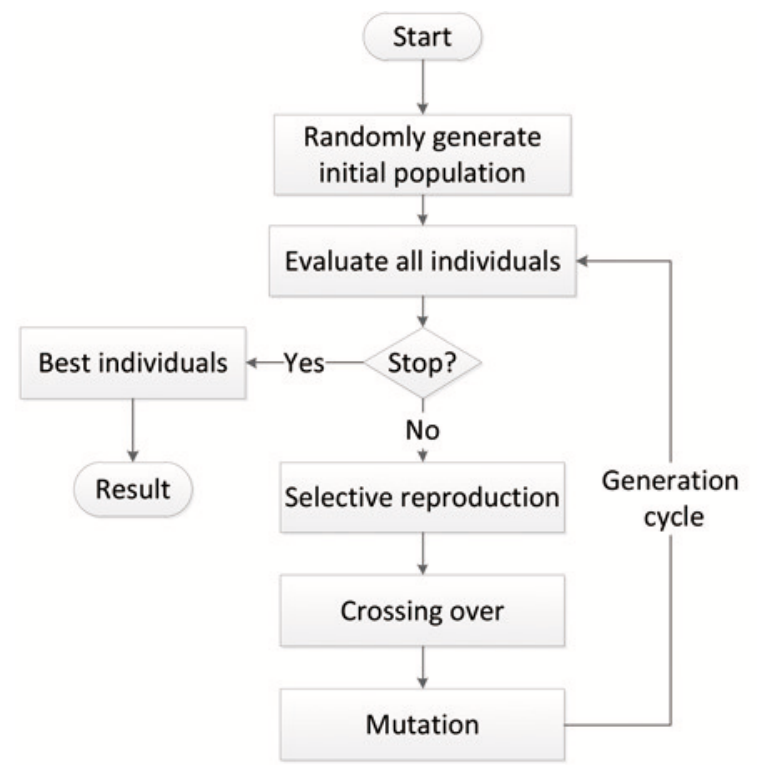

Figure A1: GA

To obtain the minimum of the objective function, it is feasible to adopt goal programming. As for the probability distribution existing in constraints or the objective function, it can be calculated after a certain amount of processing. The article designs a probabilistic planning method based on GA, which effectively resolves the proposed model (details in Fig. A2) 


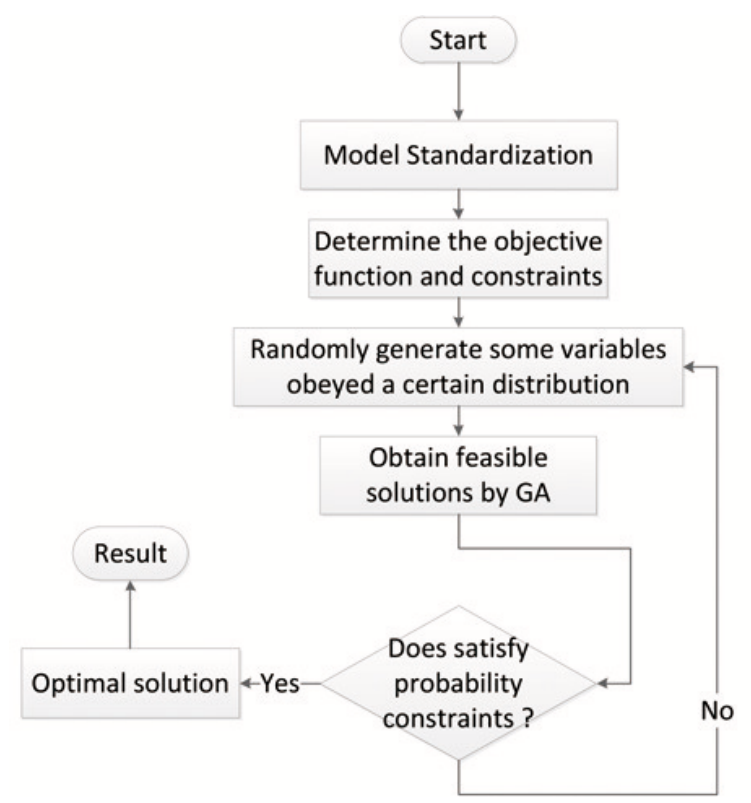

Figure A2: Probabilistic planning based on GA

\section{References}

Altuzarra, A., Moreno-Jiménez, J. M., and Sandalvador, M. (2007). A Bayesian priorization procedure for AHP-group decision making. European Journal of Operational Research, 182(1), 367-382.

Bai, J., Yang, J., Zhou, Y., and Yang, Q. (2015). A multi-step rolled forward chance-constrained model and a proactive dynamic approach for the wheat crop quality control problem. European Journal of Operational Research, 246(2), 631-640.

Ben-Arieh, D., and Easton, T. (2007). Multi-criteria group consensus under linear cost opinion elasticity. Decision Support Systems, 43(3), 713-721.

Ben-Arieh, D., Easton, T., and Evans, B. (2009). Minimum cost consensus with quadratic cost functions. IEEE Transactions on Systems Man and Cybernetics Part A: Systems and and Humans, $39(1), 210-217$.

Cabrerizo, F. J., Chiclana, F., Al-Hmouz, R., Morfeq, A., Balamash, A. S. and Herrera-Viedma, E. (2015) Fuzzy decision making and consensus: challenges. Journal of Intelligent $\mathcal{E}$ Fuzzy Systems, 29(3): 1109-1118.

Cid-Lopez, A., Hornos, M. J., Carrasco, R. A., Herrera-Viedma, E. and Chiclana, F. (2017) Linguistic multi-criteria decision-making model with output variable expressive richness. Expert Systems with Applications, 83, 350-362.

Charnes, A., and Cooper, W. W. (1959). Chance-constrained programming. Management Science, 6(1), 73-79. 
Chen, M., Wang, S. G., Wang, P. P., and Ye, X. (2016). A new equivalent transformation for interval inequality constraints of interval linear programming. Fuzzy Optimization and Decision Making, $15(2), 155-175$.

Chiclana, F., Herrera, F., and Herrera-Viedma, E. (1998). Integrating three representation models in fuzzy multipurpose decision making based on fuzzy preference relations. Fuzzy Sets and Systems, 97, (1) 3-48.

Chiclana, F., Herrera, F., and Herrera-Viedma, E. (2001). Integrating multiplicative preference relations in a multipurpose decision-making model based on fuzzy preference relations. Fuzzy Sets and Systems, 122(2), 277-291.

Chiclana, F., Tapia García, J. M., del Moral, M. J., and Herrera-Viedma, E. (2013). A Statistical Comparative Study of Different Similarity Measures of Consensus in Group Decision Making. Information Sciences, 221, 110-123.

Dai, C., Cai, Y. P., Li, Y. P., Sun, W., Wang, X. W., and Guo, H. C. (2014). Optimal strategies for carbon capture, utilization and storage based on an inexact $m_{\lambda}$-measure fuzzy chance-constrained programming. Energy, 78, 465-478.

Dong, Q., Zhü, K., and Cooper, O. (2017). Gaining consensus in a moderated group: a model with a twofold feedback mechanism. Expert Systems with Applications, 71, 87-97.

Dong, Q. X., and Cooper, O. (2016). A peer-to-peer dynamic adaptive consensus reaching model for the group AHP decision making. Ssrn Electronic Journal, 250(2), 521-530.

Dong, Y. C., Luo, N., and Liang, H. (2015). Consensus building in multiperson decision making with heterogeneous preference representation structures: A perspective based on prospect theory. Applied Soft Computing, 35, 898-910.

Dong, Y., Li, C., Chiclana, F., and Herrera-Viedma, E. (2016). Average-case consistency measurement and analysis of interval-valued reciprocal preference relations. Knowledge-Based Systems, 114, 108117.

Fishburn, P.C. (1979) Utility Theory for Decision Making, Robert E. Krieger Publishing Company.

Fodor, J.C. and Roubens, M. (1994) Fuzzy Preference Modelling and Multicriteria Decision Support, Kluwer, Dordrecht.

Forman, E., and Peniwati, K. (1998). Aggregating individual judgments and priorities with the analytic hierarchy process. European Journal of Operational Research, 108(1), 165-169. 
Fu, C., and Yang, S. L. (2010). The group consensus based evidential reasoning approach for multiple attributive group decision analysis. European Journal of Operational Research, 206(3), 601-608.

Gao, Y., and Qin, Z. (2016). A chance constrained programming approach for uncertain $p$-hub center location problem. Computers and Industrial Engineering, 102, 10-20.

Gong, Z. W., Zhang, H. H., Jeffrey, F., Li, L. S., and Xu, X. X. (2015a). Two consensus models based on the minimum cost and maximum return regarding either all individuals or one individual. European Journal of Operational Research, 240(1), 183-192.

Gong, Z. W., Xu, X. X., Zhang, H. H., Ozturk, U. A., Herrera-Viedma, E., and Xu, C. (2015b). The consensus models with interval preference opinions and their economic interpretation. Omega, 55, $81-90$.

Gong, Z. W., Xu, C., Chiclana, F., and Xu, X. X. (2017). Consensus measure with multi-stage fluctuation utility based on China's urban demolition negotiation. Group Decision and Negotiation, $26(2), 379-407$.

He, W., Chen, G., Han, Q. L., and Qian, F. (2017). Network-based leader-following consensus of nonlinear multi-agent systems via distributed impulsive control. Information Sciences, 380, 145158.

Holland, J. H. (1992). Adaptation in natural and artificial systems. MIT Press.

Honert, R. C. V. D. (1998). Stochastic group preference modelling in the multiplicative AHP: a model of group consensus. European Journal of Operational Research, 110(1), 99-111.

Hong, L. J., Yang, Y., and Zhang, L. (2011). Sequential convex approximations to joint chance constrained programs: a Monte Carlo approach. Operations Research, 59(3), 617-630.

Jiang, Y., Xu, Z., and Yu, X. (2013). Compatibility measures and consensus models for group decision making with intuitionistic multiplicative preference relations. Applied Soft Computing, 13(4), 20752086.

Kushmerick, N., Hanks, S., and Weld, D. S. (1995). An algorithm for probabilistic planning. Artificial Intelligence, 76(1-2), 239-286.

Li, C. C., Dong, Y., Herrera, F., Herrera-Viedma, E., and Martínez, L. (2017). Personalized individual semantics in computing with words for supporting linguistic group decision making. An application on consensus reaching. Information Fusion, 33(C), 29-40.

Lin, C. S., and Kou, G. (2015). Bayesian revision of the individual pair-wise comparison matrices under consensus in AHP-GDM. Applied Soft Computing, 35(C), 802-811. 
Nurmi, H. (1981) Approaches to collective decision making with fuzzy preference relations, Fuzzy Sets Syst., vol. 6, pp. 249-259.

Qin, Z. (2016). Random fuzzy mean-absolute deviation models for portfolio optimization problem with hybrid uncertainty. Applied Soft Computing, in press.

Regan, H. M., Colyvan, M., and Markovchicknicholls, L. (2006). A formal model for consensus and negotiation in environmental management. Journal of Environmental Management, 80(2), 167-176.

Saaty, T. L. (1980). The Analytic Hierarchy Process, McGraw-Hill, New York.

Sadati, M. E. H., and Nematian, J. (2013). Two-level linear programming for fuzzy random portfolio optimization through possibility and necessity-based model. Procedia Economics and Finance, 5(13), $657-666$.

Sakawa, M., and Matsui, T. (2013). Interactive fuzzy random two-level linear programming based on level sets and fractile criterion optimization. Information Sciences, 238(7), 163-175.

Srdjevic, B., and Srdjevic, Z. (2013). Synthesis of individual best local priority vectors in AHP-group decision making. Applied Soft Computing, 13(4), 2045-2056.

Srdjevic, B., Srdjevic, Z., Blagojevic, B., and Suvocarev, K. (2013). A two-phase algorithm for consensus building in AHP-group decision making. Applied Mathematical Modelling, 37(10-11), 6670-6682.

Ureña, R., Chiclana, F., Fujita, H., and Herrera-Viedma, E. (2015). Confidence-consistency driven group decision making approach with incomplete reciprocal intuitionistic preference relations. Knowledge-Based Systems, 89, 86-96.

Ureña, R., Cabrerizo, F. J., Morente-Molinera, J. A., and Herrera-Viedma, E. (2016). GDM-R: a new framework in $\mathrm{R}$ to support fuzzy group decision making processes. Information Sciences, 357, $161-181$.

Von Winterfeldt, D., and Edwards, W. (1986). Decision analysis and behavioral research. Journal of the Mount Sinai Hospital New York, 18(1), 4-14.

Wang, X., Li, Y., Ma, Z., Fan, W., Wang, L., and Xu, M. (2017). Robust optimization of structuralacoustic coupled system with random parameters. Aerospace Science and Technology, 60, 48-57.

Wang, Z. J., and Li, K. W. (2016). Group decision making with incomplete intuitionistic preference relations based on quadratic programming models. Computers and Industrial Engineering, 93(C), $162-170$. 
J. Wu and F. Chiclana. (2014a) Visual information feedback mechanism and attitudinal prioritisation method for group decision making with triangular fuzzy complementary preference relations. Information Sciences, 279, 716-734.

J. Wu and F. Chiclana. (2014b) A risk attitudinal ranking method for interval-valued intuitionistic fuzzy numbers based on novel score and accuracy expected functions. Applied Soft Computing, 22, $772-786$.

Wu, J., and Chiclana, F. (2014c). Multiplicative consistency of intuitionistic reciprocal preference relations and its application to missing values estimation and consensus building. Knowledge-Based Systems, 71, 187-200.

Wu, J., Chiclana, F. and Herrera-Viedma, E. (2015) Trust Based Consensus Model for Social Network in an Incomplete Linguistic Information Context. Applied Soft Computing, 35, 827-839.

Wu, Z., and Xu, J. (2012). A consistency and consensus based decision support model for group decision making with multiplicative preference relations. Decision Support Systems, 52(3), 757-767.

Wu, Z. B., and $\mathrm{Xu}$, J. (2016). Managing consistency and consensus in group decision making with hesitant fuzzy linguistic preference relations. Omega, 65(3), 28-40.

Wu, Y. N., Xu, H., Xu, C., and Chen, K. (2016). Uncertain multi-attributes decision making method based on interval number with probability distribution weighted operators and stochastic dominance degree. Knowledge-Based Systems, 113, 199-209.

Xu, Z. S., and Liao, H. (2015). A survey of approaches to decision making with intuitionistic fuzzy preference relations. Knowledge-Based Systems, 80(C), 131-142.

Xu, X. H., Zhong, X. Y., Chen, X. H., and Zhou, Y. J. (2015). A dynamical consensus method based on exit-delegation mechanism for large group emergency decision making. Knowledge-Based Systems, 86(C), 237-249.

Zadeh,L. A. (1975) The concept of a linguistic variable and its applications to approximate reasoning. Part I. Information Sciences, 8:199-249.

Zhang, B., Dong, Y., and Xu, Y. (2013). Maximum expert consensus models with linear cost function and aggregation operators. Computers and Industrial Engineering, 66(1), 147-157.

Zhang, F., Ignatius, J., Zhao, Y., Lim, C. P., Ghasemi, M., and Ng, P. S. (2015). An improved consensus-based group decision making model with heterogeneous information. Applied Soft Computing, 35, 850-863.

Zhao, L., Jia, Y., Yu, J., and Du, J. (2017). H $\infty$ sliding mode based scaled consensus control for linear multi-agent systems with disturbances. Applied Mathematics and Computation, 292, 375-389. 